\title{
SEGUIMIENTO ENDOSCÓPICO E HISTOLÓGICO POST ABLACIÓN CON ARGÓN PLASMA DEL ESÓFAGO DE BARRETT EXTENSO EN PACIENTES SOMETIDOS A TRATAMIENTO QUIRÚRGICO PREVIO*
}

\author{
Drs. Italo Braghetto M. ${ }^{1}$, Héctor Valladares H. ${ }^{1}$, Danny Oksenberg R. ${ }^{2}$, \\ Ints. Felipe Sánchez T. ${ }^{3}$, Christian Ramos S. ${ }^{3}$ \\ 1 Departamento de Cirugía. \\ 2 Departamento de Medicina. \\ 3 Internos de Medicina, Facultad de Medicina. \\ Hospital Clínico Universidad de Chile. \\ Santiago, Chile.
}

\begin{abstract}
Argon plasma ablation of extensive Barrett esophagus. Experience in 19 patients

Background: Endoscopic argon plasma ablation of Barrett esophagus decreases the risk of future esophageal cancer development. Aim: To assess the endoscopic regression of columnar epithelium and the presence of intestinal metaplasia among patients operated for Barrett esophagus and subjected to argon plasma ablation. Patients and Methods: In 19 patients with extensive Barrett esophagus subjected to a Nissen fundoplication, Barrett esophagus was endoscopically ablated with argon plasma. Patients were assessed 6 and 12 months after surgery to evaluate the regression of columnar epithelium and the presence of intestinal metaplasia. Results: One, two and three ablation sessions were carried out in 10, three and six patients, respectively. Three patients had complications. The initial length of columnar epithelium segment was $52 \pm 15.6$ $\mathrm{mm}$ and decreased to $22.6 \pm 10.6 \mathrm{~mm}(\mathrm{p}<0.05)$. In 12 patients, there was absence of intestinal metaplasia on follow up, in six it persisted (one of them with "buried cells") and in one patient, dysplasia appeared. Conclusions: Endoscopic argon plasma ablation may have a complementary therapeutic role for the regression of columnar epithelium in Barrett esophagus.
\end{abstract}

Key words: Barrett esophagus, argon plasma ablation, Nissen fundoplication.

\section{Resumen}

Introducción: Pacientes con esófago de Barrett extenso presentan un riesgo de cáncer. De allí surge la posibilidad de someter a estos pacientes a ablación con argón plasma por vía endoscópica para disminuir este riesgo de desarrollar un adenocarcinoma. Objetivo: Evaluar la regresión endoscópica del epitelio columnar

*Recibido el 4 de abril de 2014 y aceptado para publicación el 12 de mayo de 2014.

Los autores no refieren conflictos de interés.

Correspondencia: Dr Italo Braghetto M.

ibraghet@hcuch.cl 
y la presencia de metaplasia intestinal en pacientes operados por esófago de Barrett y sometidos a ablación con argón plasma. Material y Método: Se incluyen 19 pacientes en este estudio, todos ellos con esófago de Barrett extenso confirmado por endoscopia e histología, los cuales se someten a ablación con argón plasma por vía endoscópica. Estos pacientes se controlaron con endoscopia e histológicamente a los 6 meses y al año de operados para evaluar la regresión del área con epitelio columnar y precisar la presencia histológica de metaplasia intestinal. Resultados: En 10 pacientes se efectuó 1 sesión de ablación, en 3 pacientes 2 sesiones y en 6 pacientes se efectuó 3 sesiones de ablación. Tres pacientes presentaron complicaciones. El largo de las lengüetas de epitelio columnar inicial fue de $52 \pm 15,6 \mathrm{~mm}$ el cual disminuyó a $22,6 \pm 10,6 \mathrm{~mm}(\mathrm{p}<0,05)$. El seguimiento histológico reveló ausencia de metaplasia intestinal en 12 pacientes $(63,1 \%)$ persistencia de metaplasia en 6 pacientes ( 1 de ellos con células en submucosa, "buried cells") y un paciente con aparición de displasia. Conclusión: La ablación con argón plasma puede tener un rol en el tratamiento complementario a la cirugía para mejorar la regresión del epitelio columnar y disminuir los riesgos de presentar un adenocarcinoma de Barrett.

Palabras clave: Esófago de Barrett, endoscopia, ablación.

\section{Introducción}

El esófago de Barrett (metaplasia intestinal en esófago distal) es considerado un factor de riesgo para el desarrollo de adenocarcinoma de esófago, entre un $0,4 \%$ a $1 \%(250-410$ pacientes/año post tratamiento) y se asocia con un riesgo 30-60 veces mayor de desarrollar adenocarcinoma del esófago en comparación con la población general. El tratamiento de la enfermedad de reflujo gastroesofágico (ERGE), ya sea médico o con fundoplicatura de Nissen, ha demostrado ser ineficaz en la eliminación o incluso la reducción del riesgo de cáncer. Por ende, actualmente el tratamiento se ha centrado en la ablación de la mucosa metaplásica, combinándolo con un tratamiento médico o quirúrgico de la ERGE, para evitar el desarrollo de adenocarcinoma de esófago.

En pacientes con esófago de Barrett (EB) sin displasia, los resultados de los tratamientos ablativos en los períodos de seguimiento de hasta 6,8 años han sido prometedores. De allí surge la posibilidad de someter a estos pacientes a ablación con argón plasma por vía endoscópica para disminuir este riesgo de desarrollar un adenocarcinoma ${ }^{1-5}$. Recientemente se ha descrito la restauración parcial de la mucosa escamosa después de la ablación de EB con técnicas endoscópicas ${ }^{6,7}$.

\section{Objetivo}

Evaluar la regresión endoscópica del epitelio columnar y de la presencia de metaplasia intestinal en pacientes operados por esófago de Barrett y sometidos a ablación con argón plasma.

\section{Material y Método}

Se incluyen 19 pacientes en este estudio, todos ellos con esófago de Barrett extenso confirmado por endoscopia e histología preoperatoria, en los cuales se demostró la presencia de metaplasia intestinal de diferente extensión. Estos pacientes se someten a ablación con argón plasma (AP) (Figura 1) por vía endoscópica bajo anestesia general después de haber sido sometidos a cirugía antirreflujo con Fundoplicatura de Nissen sola (6 pacientes) o combinado con cirugía de supresión ácida y derivación duodenal para evitar reflujo biliar (13 pacientes) de acuerdo a la extensión del epitelio columnar sea corto $(<5 \mathrm{~cm})$ o extenso $(>5 \mathrm{~cm})$ de longitud de las lengüetas o del área de epitelio metaplásico ${ }^{8}$. El criterio para escoger una u otra técnica antirreflujo, se debió a:

- El largo de la mucosa con metaplasia intestinal (Esófago de Barrett).

- Existencia o ausencia de complicaciones del Esófago de Barrett.

- Solicitud del paciente.

Los plazos entre la cirugía inicial y la terapia endoscópica oscilan entre 2 y 8 años, para los 19 casos (promedio 5,5 años). Todos estos pacientes tuvieron seguimiento endoscópico periódico anual y en vista de la no regresión del epitelio columnar se les ofreció esta terapéutica complementaria dando su consentimiento informado.

Para corroborar el éxito de la cirugía inicial, todos los sujetos del presente estudio fueron sometidos a un análisis manométrico y a un monitoreo de $\mathrm{pH}$ intraesofágico de $24 \mathrm{~h}$. La presión del esfínter gastroesofágico (EGE) normal es $18 \pm 6 \mathrm{mmHg}$, y se consideró una presión menor que $10 \mathrm{mmHg}$ para definir un esfínter hipotensivo. Se consideró un EGE incompetente cuando cumplió al menos con una de las siguientes características: presión de reposo menor a $6 \mathrm{mmHg}$, largo total menor a $2 \mathrm{~cm}$ y largo abdominal menor a $10 \mathrm{~mm}$.

El monitoreo de $\mathrm{pH}$ intraesofágico de $24 \mathrm{~h}$ se realizó inmediatamente después del estudio manométrico, una vez identificada la exacta ubicación del 

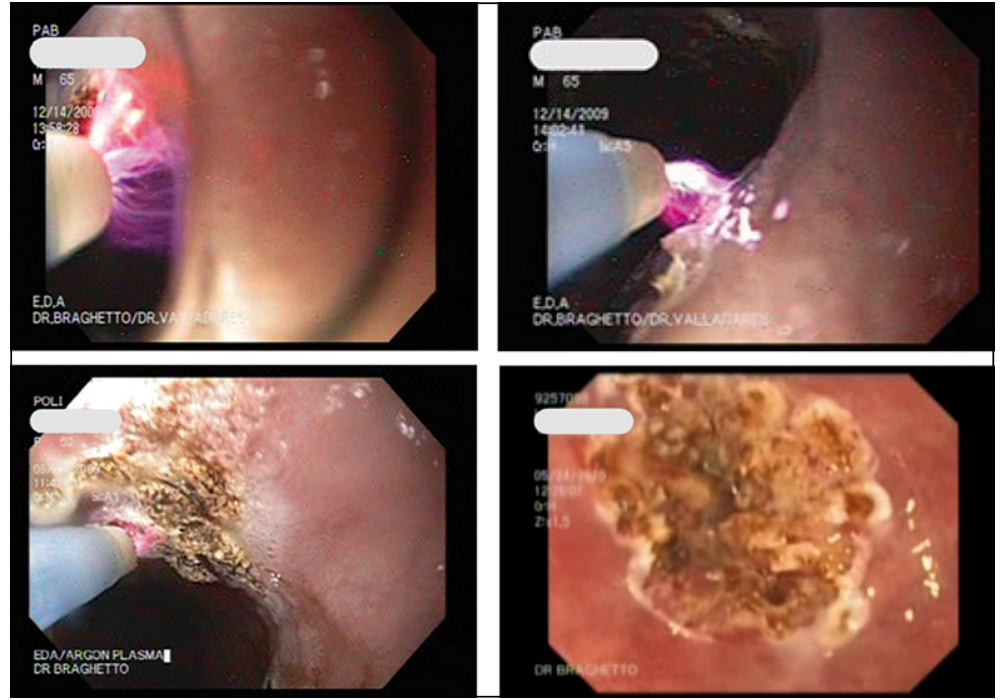

Figura 1. Aplicación endoscópica de coagulación con argón plasma: resultados inmediatos.

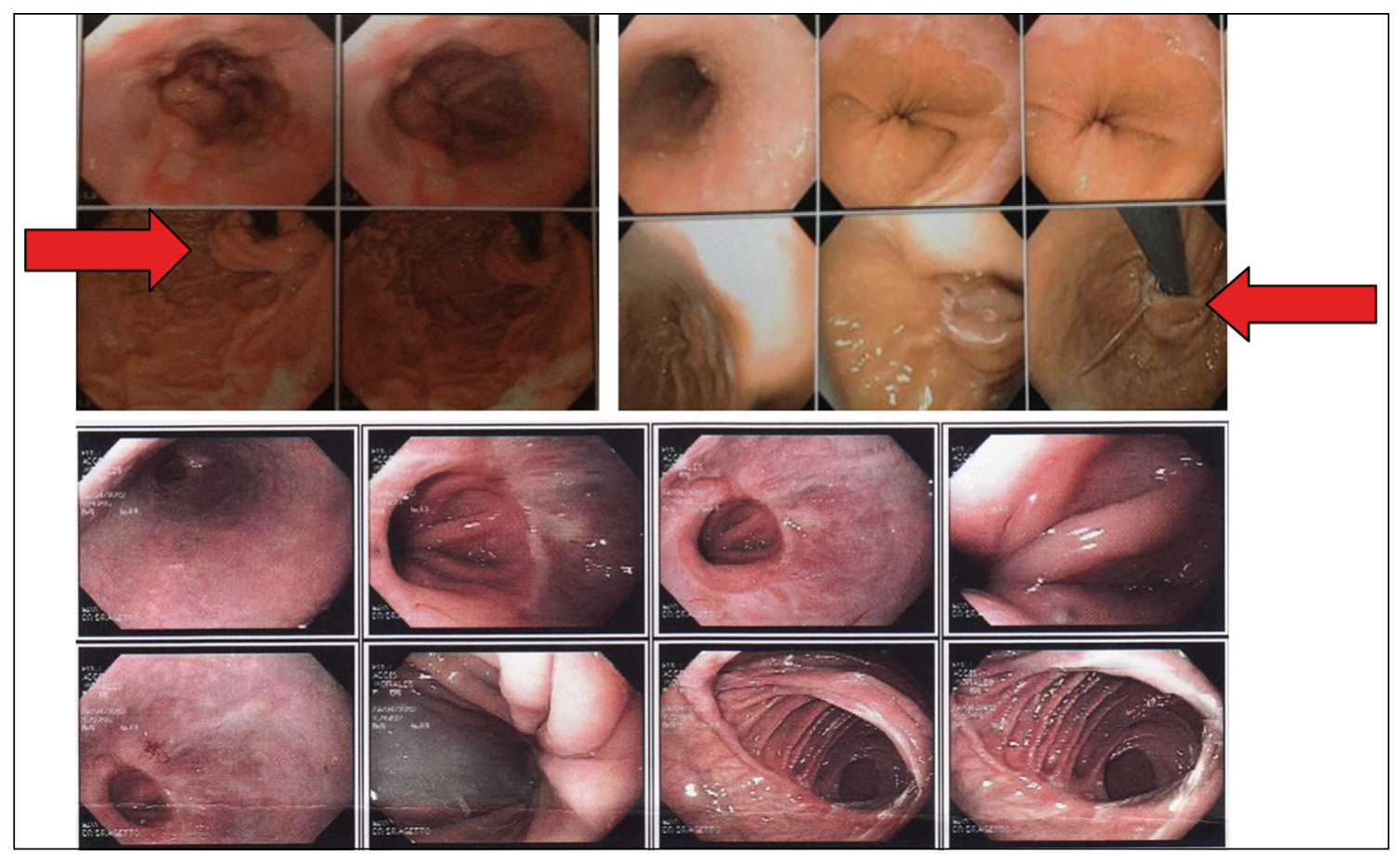

Figura 2. Control endoscópico post aplicacion de coagulación con argón plasma: Resultados tardíos demostrando regresion de las lengüetas de epitelio columnar, reepitelización con epitelio escamoso y en la imagen con retrovisión endoscópica, se puede apreciar un cardias tipo I de Hill (flecha).

esfínter gastroesofágico. Para el presente estudio se consideró una exposición ácida $(\mathrm{pH}<4)$ del esófago distal mayor al $4 \%$ durante las $24 \mathrm{~h}$ como un RGE patológico.

Endoscópicamente todos los pacientes tenían en el preoperatorio un cardias dilatado tipo III de Hill y luego de la cirugía se confirmó una buena válvula antirreflujo (cardias tipo I según la nomenclatura difundida por Hill) (Figura 2, flechas).

El número de sesiones necesarias para conseguir ablación depende de la respuesta clínica y endoscópica observada en los controles endoscópicos sucesivos, que en promedio son 3 por paciente, a los 3, 6 meses y al año de sometidos al procedimiento. 
Los pacientes quedan durante 1 mes con inhibidores de bomba de protones (IBP). Estos pacientes se controlaron con endoscopia e histológicamente a los 6 meses y al año de operados para determinar la regresión del área del epitelio columnar.

Los 19 pacientes tenían consentimiento informado para el procedimiento y el estudio fue aprobado por el comité de ética y OAIC (Oficina de Apoyo a la Investigación Clínica) de nuestro hospital.

Para el análisis de los resultados, se empleó el promedio de la longitud de las lengüetas con desviación estándar de cada individuo en su etapa inicial y final del tratamiento. La disminución promedio de las lengüetas se calculó en base a la diferencia del largo final menos el largo inicial de cada paciente y su respectiva desviación estándar. Complementariamente se compara cada disminución individual de las lengüetas con la disminución promedio de la muestra. Para el cálculo de significación estadística se usó el método "t" de Student.

\section{Resultados}

El análisis manométrico realizado post cirugía, mostró un esfínter competente para los 19 pacientes, con valores superiores a los del preoperatorio. En general para la muestra la presión promedio del EGE pre-operatorio fue de 7,6 $\pm 2,7 \mathrm{mmHg}$ y el promedio de la $\mathrm{pH}$ metria pre-cirugía fue de $13,7 \pm 2,9 \%$ de reflujo ácido en $24 \mathrm{~h}$ con un score DeMeester de 43,8 $\pm 20,1$. En el postoperatorio se confirmó un aumento de la presión del esfínter esofágico inferior a una presión de $16,3 \pm 3,8 \mathrm{mmhg}$ $(\mathrm{p}<0,05)$ y phmetría de $3,1 \pm 0,3 \%$ en 24 h y score DeMeester de 11,9 $\pm 6,7 p<0,01$, comparado con el preoperatorio). Se observó que 3 pacientes de los 19 estudiados, si bien disminuyeron sus índices de reflujo en el postoperatorio, presentaron valores sobre lo normal, lo que da cuenta de la efectividad del procedimiento quirúrgico independiente del tipo de cirugía efectuada. Dado estos hallazgos se consideró innecesaria la separación de los resultados tomando en cuentan el tipo de cirugía efectuada.

La Figura 2 muestra el control endoscópico alejado que se observa más frecuentemente en los pacientes sometidos a ablación con argón plasma confirmando la disminución del largo de las lengüetas de epitelio columnar. En 2 ocasiones se observó un límite neto nacarado del cambio de mucosas con estenosis que necesitó de dilataciones endoscópicas.

En la Tabla 1 se muestra el número de sesiones que se necesitaron para lograr una regresión significativa de la extensión del área con mucosa columnar. La gran mayoría de los pacientes fueron sometidos sólo a una sesión. Sin embargo, esto obedece a que la extensión del epitelio columnar era más bien de tipo lengüetiforme. En el resto de los pacientes se necesitó más de una sesión dado que el área de epitelio columnar era más extenso y con lengüetas más anchas.

La Tabla 2 muestra las complicaciones observadas en este estudio, destacando un caso con úlcera esofágica y 2 casos de estenosis que debieron ser sometidas a dilataciones endoscópicas con bujías de Savary en varias ocasiones. El dolor retrosternal estuvo presente en todos los casos, de intensidad leve a mediano ( 3 a 6 de 10 en la escala de dolor) que fue manejado con omeprazol (40 mg cada $12 \mathrm{~h}$ por vía oral) y ketoprofeno por vía intravenosa con lo cual el dolor cede en un plazo de 2 a 6 días. Los pacientes se mantuvieron con inhibidores de la bomba de protones al menos por 2 meses.

En la Tabla 3 se muestran los resultados histológicos obtenidos a los 6 meses post aplicación

Tabla 1. Ablación con Argón plasma: número de sesiones necesarias para obtener regresión del área de mucosa columnar $(n=19)$

\begin{tabular}{|ccc|}
\hline $\mathbf{n}$ & $\mathbf{n}$ sesiones & \% de la muestra \\
14 & 1 & 77,7 \\
1 & 2 & 5,5 \\
4 & 4 & 16,6 \\
\hline
\end{tabular}

Tabla 2. Complicaciones precoces y tardías post ablación de coagulación con argón plasma por vía endoscópica $(n=19)$

\begin{tabular}{|lrc|}
\hline Complicaciones & n & \% de la muestra \\
Úlcera esofágica & 1 & 5,55 \\
Estenosis & 2 & 11,11 \\
Dolor torácico & 19 & 100 \\
\hline
\end{tabular}

Tabla 3. Seguimiento histológico a los 6 meses post aplicación endoscópica de argón plasma: presencia de metaplasia intestinal $(n=19)$

\begin{tabular}{|ll|}
\hline Histología & n (\%) \\
$\begin{array}{l}\text { Ausencia de metaplasia intestinal } \\
\text { (en las muestras tomadas) }\end{array}$ & $12(63,1 \%)$ \\
Persistencia de metaplasia & $7(36,8 \%)^{*}$ \\
& $\begin{array}{l}1 \text { con células en submu- } \\
\text { cosa, "buried cells" } \\
1 \text { paciente con aparición } \\
\text { de displasia }\end{array}$ \\
\hline
\end{tabular}

*4 en espera de re-tratamiento con radiofrecuencia. 


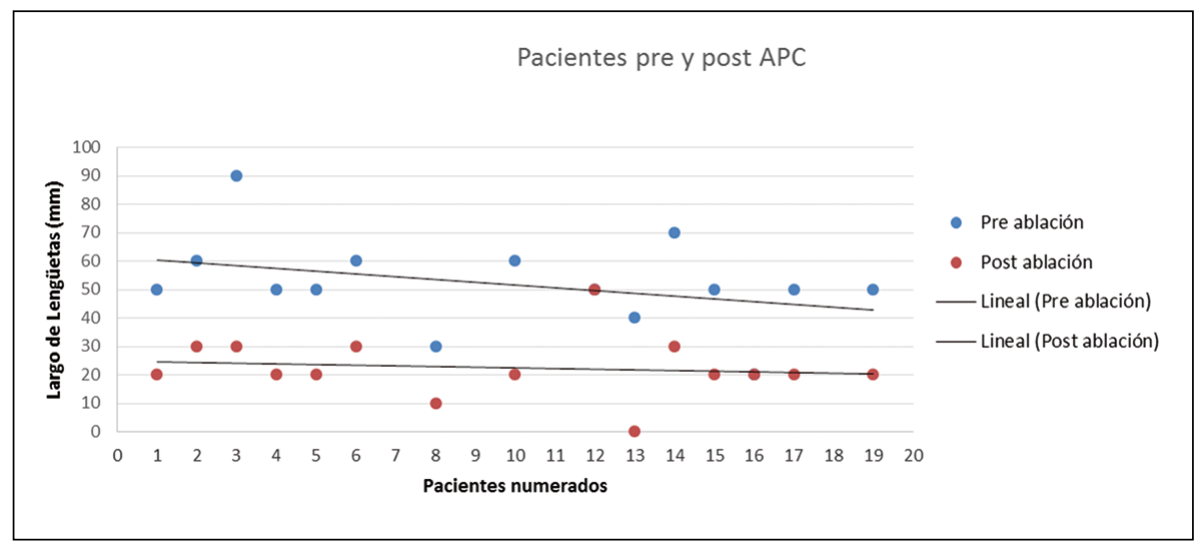

Figura 3. Gráfico de los pacientes pre y post coagulación con Argón Plasma (APC). Inicial $=52 \pm 15,6$ mm. Final $=22,6 \pm$ $10,6 \mathrm{~mm}(\mathrm{p}<0,05)$. Disminución promedio $=29,3 \pm 19,6 \mathrm{~mm}$.

de argón plasma, observando ausencia de epitelio columnar en el 63\% de los pacientes en al menos 6 muestras tomadas para biopsia de la zona de ablación; pero es notable que un tercio de los pacientes presentaban islas de epitelio columnar remanentes o persistencia de epitelio de Barrett, destacando en 1 paciente células caliciformes bajo el epitelio escamoso y en otro caso progresión a displasia. Uno de estos pacientes fue nuevamente sometido a ablación con argón plasma y el resto está en espera de ser sometidos a tratamiento con radiofrecuencia.

En la Figura 3 se muestra la disminución del largo de las lengüetas de epitelio columnar. El largo inicial observado fue de $52,15 \pm 15,6 \mathrm{~cm}$ y el largo final logrado fue de $22,6 \pm 10,6 \mathrm{~mm}(\mathrm{p}<0,05)$ con una disminución promedio de casi $3 \mathrm{~cm}$ del largo del área de Barrett.

\section{Discusión}

Se ha demostrado que los pacientes con metaplasia de Barrett tienen de 30 a 125 veces mayor riesgo de desarrollar adenocarcinoma de esófago (1\% de los pacientes) siendo el rango de aparición anual de adenocarcinoma de Barrett de 1 paciente cada 44 a 255 pacientes por año de seguimiento ${ }^{9}$. De allí surge la necesidad de destruir el epitelio metaplásico y regenerarlo con epitelio escamoso eliminando así el daño provocado por la presencia de reflujo ácido y/o biliar mediante tratamiento médico con inhibidores de la bomba de protones o mediante algún procedimiento quirúrgico. Esta terapia de combinación puede convertirse en el estándar en la prevención de adenocarcinoma de esófago secundario al esófago de Barrett.

En la experiencia de varios autores, la coagulación con Argón plasma (APC) en combinación con la cirugía antirreflujo es una técnica eficaz y segura para la ablación completa de la mucosa metaplásica y no displásica del epitelio de Barrett. Esto debido a que la ablación podría reducir o retrasar el riesgo de aparición de cáncer; los datos recientes, aunque no definitivos, sugieren beneficio.

En la actualidad, diversos métodos de tratamiento están disponibles para la ablación de la mucosa metaplásica del esófago, ya sea mediante ablación por radiofrecuencia o ablación con aplicación de argón plasma. Las diferencias entre las diversas técnicas probablemente se desprenden de la facilidad de aplicación, su eficacia en el logro completo de la ablación, y la estabilidad del epitelio escamoso regenerado. La mucosa neo-escamosa, regenerada post ablación de la mucosa de Barrett más el tratamiento de la ERGE con fundoplicatura, se mantiene estable durante la evaluación de seguimiento a largo plazo en dos tercios de los pacientes con erradicación inicial $^{8-12}$. Los estudios prospectivos respecto de la eficacia de un enfoque multimodal, consistentes en la ablación con AP combinada con fundoplicatura laparoscópica para el tratamiento del esófago de Barrett, han mostrado que el epitelio escamoso es restaurado por completo en todos los pacientes. La tasa de éxito de la ablación después de la cirugía laparoscópica antirreflujo puede ser tan alta como $80-91 \%$. Sin embargo, en algunos pacientes pueden persistir pequeñas islas de metaplasia intestinal bajo el nuevo epitelio escamoso. En el 68,4\% de los casos se requieren de más de una sesión de ablación, las complicaciones son poco comunes y generalmente leves, siendo la más frecuente las molestias $\mathrm{y}$ dolor retrosternal que generalmente duran pocos días, pero en otros pueden persistir durante un período más prolongado, lo que requiere medicación analgésica. La disfagia y odinofagia de corta duración se observan en casi un $25 \%$ de los casos. En estas circunstancias, los autores recomiendan que la ablación endoscópica del epitelio de Barrett debiera limitarse a los ensayos clínicos controlados ${ }^{6}$. 
Hay 4 estudios que evalúan los resultados de argón plasma versus el tratamiento fotodinámico (TFD) demostrando que el AP no tuvo efectos para erradicar completamente la mucosa de Barrett y la TDF no fue efectiva en eliminar displasia. La ablación completa se logró en un $59 \%$ con AP versus $28 \%$ con TDF $(\mathrm{p}<0,008)$. La incidencia de glándulas enterradas fue de 19 a $52 \%$ con AP versus 5 a $11 \%$ con TFD.

En algunos reportes se ha observado que la regresión de la extensión del esófago de Barrett tras fundoplicatura es más probable en los pacientes que se someten a la ablación con Argón plasma comparado a los pacientes que no fueron operados previamente. En la mayoría de los pacientes tratados con ablación la mucosa neo-escamosa se mantiene estable hasta 5 años de seguimiento y el desarrollo de la displasia de alto grado sólo ha ocurrido en los pacientes que no han sido tratados con argón plasma ${ }^{10,11}$. Por lo tanto, la ablación con argón plasma puede tener un rol como tratamiento complementario a la cirugía para mejorar la regresión del epitelio columnar y disminuir los riesgos de presentar adenocarcinoma de Barrett, siempre y cuando se logre un adecuado control del reflujo y normalidad del monitoreo de $\mathrm{pH}$ en el postoperatorio ${ }^{12}$. En las series reportadas la endoscopia post-operatoria fue normal en más del $80 \%$ de los casos pero a largo plazo la efectividad de la cirugía antirreflujo va disminuyendo ${ }^{8}$, por lo que en estos pacientes se debe instaurar un tratamiento con inhibidores de la bomba de protones o efectuar definitivamente una cirugía de supresión ácida y derivación biliar, que es la técnica que nosotros preconizamos como la cirugía primaria de elección en esófago de Barrett extenso o complicado, asegurándonos un control de todo tipo de reflujo sea este ácido o biliar.

Los estudios han mostrado que la reducción de las lengüetas de epitelio columnar es muy similar a la observada en nuestra experiencia (mediana de $3,6 \mathrm{~cm}$, con un rango de $1 \mathrm{a} 6 \mathrm{~cm}$ ) y el número de sesiones para obtener estos resultados reportada en a literatura es en promedio de 4,9 sesiones (rango de 1-16) definiendo a la erradicación del epitelio de Barrett como la conversión histológica de la mucosa intestinal a epitelio escamoso.

No se han reportado cambios displásicos, pero ha sido comunicada la persistencia de islotes de la mucosa columnar subyacente o células glandulares sub-enterrados ${ }^{13}$. En otros estudios de seguimiento a largo plazo, la tasa de recaída y presencia de metaplasia intestinal después de la ablación del Barrett complementaria al tratamiento quirúrgico, ha oscilado entre el $11 \%$ y $60 \%$ a los 5 años ${ }^{14}$. Por lo tanto, la eliminación del epitelio metaplásico o displásico y la continua reducción del reflujo tanto ácido como biliar es crucial para la prevención de la progresión del esófago de Barrett, progresión a displasia y adenocarcinoma esofágico ${ }^{15,16}$.

Más recientemente la ablación con radiofrecuencia endoluminal endoscópica está ganando aceptación como un medio para eliminar el esófago de Barrett y se ha demostrado ser eficaz en diversas etapas de la enfermedad con los mínimos efectos secundarios $\mathrm{y}$, por lo tanto, reducir el riesgo de desarrollar adenocarcinoma de esófago. De hecho, la reciente revisión Cochrane sobre el tratamiento para el esófago de Barrett con la ablación por radiofrecuencia, señala que puede convertirse en el tratamiento de elección para pacientes con esófago de Barrett aún sin displasia ${ }^{16,17}$. En nuestros pacientes con persistencia de la metaplasia intestinal después de argón plasma hemos efectuado ablación con radiofrecuencia al disponer actualmente de esta tecnología en nuestro departamento.

En conclusión, la ablación con argón plasma puede tener un rol en el tratamiento complementario a la cirugía para mejorar la regresión del epitelio columnar y disminuir los riesgos de presentar un adenocarcinoma de Barrett. Sin embargo, tiene la gran limitación que representa la necesidad de varias sesiones para lograr ablación completa.

\section{Referencias}

1. Kauttu T, Räsänen J, Krogerus L, Sihvo E, Puolakkainen P, Salo JA. Long-term results of ablation with antireflux surgery for Barrett's esophagus: a clinical and molecular biologic study. Surg Endosc. 2012;26:1892-7.

2. Tigges H, Fuchs KH, Maroske J, Fein M, Freys SM, Müller J, et al. A Combination of endoscopic argon plasma coagulation and antireflux surgery for treatment of Barrett's esophagus. J Gastrointest Surg. 2001;5:251-9.

3. Lenglinger J, Riegler M. Radiofrequency ablation if Barrett's esophagus persists after fundoplication? Surg Endosc. 2010;24:2363-4.

4. Morris CD, Byrne JP, Armstrong GR, Attwood SE. Antireflux surgery does not remove cancer risk in Barrett's esophagus Prevention of the neoplastic progression of Barrett's oesophagus by endoscopic argon beam plasma ablation. Br J Surg. 2001;88:1357-62.

5. Bonavina L, Ceriani C, Carazzone A, Segalin A, Ferrero S, Peracchia A. Endoscopic laser ablation of nondysplastic Barrett's epithelium: is it worthwhile? J Gastrointest Surg. 1999;3:194-9.

6. Morino M, Rebecchi F, Giaccone C, Taraglio S, Sidoli L. Endoscopic ablation of Barrett's esophagus using argon plasma coagulation (APC) following surgical laparoscopic fundoplication. Surg Endosc. 2003;17:53942.

7. Ragunath K, Krasner N, Raman VS, Haqqani MT, Phi- 
llips CJ, Cheung I. Endoscopic ablation of dysplastic Barrett's oesophagus comparing argon plasma coagulation and photodynamic therapy: a randomized prospective trial assessing efficacy and cost-effectiveness. Scand J Gastroenterol. 2005;40:750-8.

8. Csendes A, Burdiles P, Braghetto I, Korn O, Díaz JC, Rojas J. Early and late results of the acid suppression and duodenal diversion operation in patients with Barrett's esophagus: analysis of 210 cases. World J Surg. 2002;26:566-76.

9. Pinotti AC, Cecconello I, Filho FM, Sakai P, GamaRodrigues JJ, Pinotti HW. Endoscopic ablation of Barrett's esophagus using argon plasma coagulation: a prospective study after fundoplication. Dis Esophagus. 2004; 17:243-6

10. Franchimont D, Van Laethem JL, Devière J. Gastrointest Argon plasma coagulation in Barrett's esophagus. Endosc Clin N Am. 2003;13:457-66.

11. Bright T, Watson DI, Tam W, Game PA, Astill D, Ackroyd R, et al. Randomized trial of argon plasma coagulation versus endoscopic surveillance for Barrett esophagus after antireflux surgery: late results. Ann Surg. 2007;246:1016-20.

12. Formentini A, Schwarz A, Straeter J, Stanescu A,
Henne-Bruns D. Treatment of Barrett's esophagus with argon plasma coagulation and antireflux surgery. A retrospective analysis. Hepatogastroenterology 2007;54:1991-6.

13. Lenglinger J, Riegler M Radiofrequency ablation and dilated columnar-lined esophagus. Am J Gastroenterol. 2009;104:2631.

14. Hubbard N, Velanovich V. Endoscopic endoluminal radiofrequency ablation of Barrett's esophagus in patients with fundoplications. Surg Endosc. 2007;21:625-8.

15. Shaheen NJ, Kim HP, Bulsiewicz WJ, Lyday WD, Triadafilopoulos G, Wolfsen HC, et al. Prior fundoplication does not improve safety or efficacy outcomes of radiofrequency ablation: results from the U.S. RFA Registry. J Gastrointest Surg. 2013;17:21-29.

16. O'Connell K, Velanovich V. Effects of Nissen fundoplication on endoscopic endoluminal radiofrequency ablation of Barrett's esophagus. Surg Endosc. 2011;25:8304.

17. Bowers SP, Mattear SG, Waring PJ, Galloway K, Nasir A, Pascal R, et al. KTP (potassium-titanyl-phosphate) laser ablation of Barrett's esophagus after anti-reflux surgery results in long-term loss of intestinal metaplasia. Surg Endosc. 2003;17:49-54. 